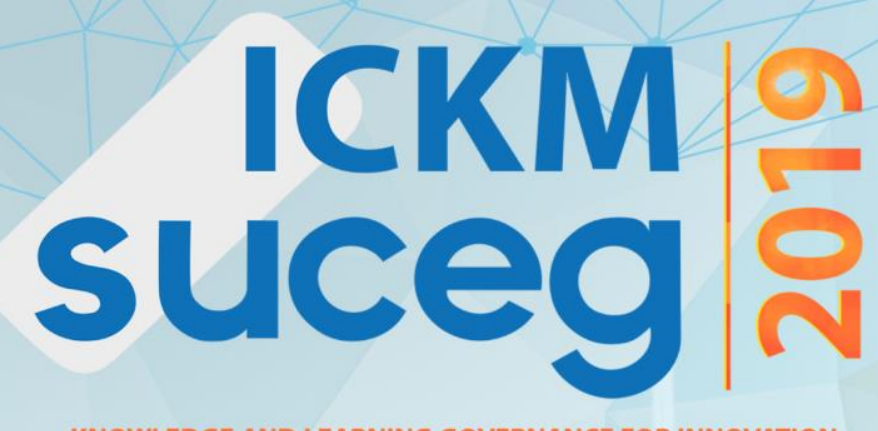

\title{
POSSÍVEIS DIRETRIZES PARA A GAMIFICAÇÃO COM BASE EM FERRAMENTAS DE DESIGN THINKING
}

Camila Menegali

Bacharel em Design pela Universidade Federal de Santa Catarina, https://orcid.org/0000-00017594-0468,menegalicamila@gmail.com

Aline Debize de Fraga

Tecnóloga em Gestão de Recursos Humanos pelo Instituto de Ensino Superior da Grande Florianópolis, https://orcid.org/0000-0003-4187-7223, debize.aline@gmail.com

Maria Collier de Mendonça

Bolsista CAPES PNPD, Pós-Doutoranda no Programa de Pós-Graduação em Engenharia e Gestão do Conhecimento na Universidade Federal de Santa Catarina, https://orcid.org/0000-0003-23524520, mariacmendonca@gmail.com

Richard Perassi Luiz de Sousa

Professor Titular no Programa de Engenharia e Gestão do Conhecimento da Universidade

Federal de Santa Catarina, https://orcid.org/0000-0003-0696-4110, richard.perassi@gmail.com

Francisco Antônio Pereira Fialho

Professor Titular no Programa de Engenharia e Gestão do Conhecimento da Universidade Federal de Santa Catarina, https://orcid.org/0000-0001-6826-7180, fapfialho@gmail.com

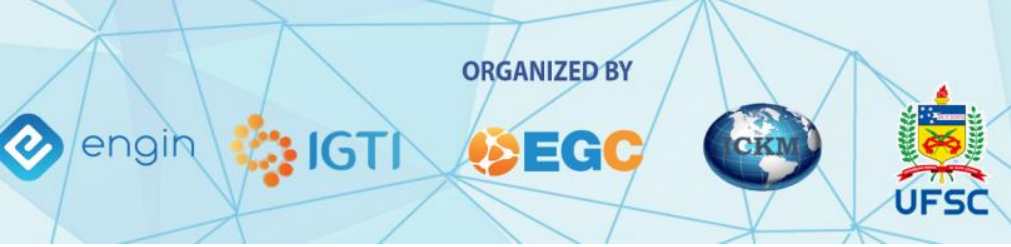




\section{RESUMO}

Objetivo: Este trabalho propõe a discussão de possíveis diretrizes para aplicação do design thinking em múltiplos contextos de gamificação.

Design/Metodologia/Abordagem: A metodologia utilizada envolveu uma revisão narrativa de literatura sobre os temas gamificação e design thinking; seguida por uma análise interpretativa dos pontos de intersecção entre os dois temas.

Resultados: A gamificação explora os potenciais de motivação e envolvimento dos jogos em contextos não relacionados a eles, enquanto o design thinking é uma abordagem voltada à solução de problemas de maneiras inovadoras e inspiradas no design. Foram explorados os modos como o design thinking pode contribuir para os processos de gamificação em diferentes situações, dentre eles o conhecimento do público-alvo e a prototipação das estratégias de gamificação.

Limitações da pesquisa: Identificou-se a existência de poucas publicações acadêmicas dedicadas à aplicação do design thinking em projetos aplicados ao desenvolvimento de gamificação para distintos ambientes.

Originalidade/valor: O artigo expande os estudos sobre o tema pesquisado, podendo contribuir para futuras pesquisas sobre diretrizes para gamificação a serem aplicadas em variados ambientes.

Palavras-chave: Gamificação. Design thinking. Inovação. 


\title{
POSSIBLE GUIDELINES FOR GAMIFICATION BASED ON DESIGN THINKING TOOLS
}

\begin{abstract}
Goal: This paper proposes to develop guidelines for gamification in multiple contexts based on design thinking.

Design / Methodology / Approach: The methodology involved a narrative literature review on gamification and design thinking; followed by an interpretive analysis of the intersections between both themes.

Results: Gamification explores potential motivations and engagement within the games or nongaming contexts, while design thinking is an innovative, and design-inspired problem-solving approach. We have explored how design thinking may contribute to gamification processes in different situations, such as getting to know the target audience and the prototyping of gamification strategies.
\end{abstract}

Limitations of the research: We identified there are few academic publications dedicated to the application of design thinking or applied projects aimed at the development of gamification for distinct environments.

Originality/value: This paper expands studies of the theme investigated, and it may also contribute to future studies on guidelines for gamification in different environments.

Keywords: Gamification. Design thinking. Innovation. 


\section{INTRODUÇÃO}

Os jogos estão presentes em nossa cultura desde o início dos tempos, segundo Huizinga (2000) os jogos são ainda mais antigos do que a cultura, pois elementos dos jogos humanos estão presentes até mesmo nas brincadeiras dos animais. Um dos mais antigos exemplos de jogos que temos conhecimento, o Jogo Real de Ur, tem sua idade estimada em 4.500 anos (Neto, 2016). Desde então este tipo de entretenimento tem adquirido cada vez mais importância em nossa sociedade, principalmente com o advento dos videogames, que mudaram os modos como as pessoas jogam. Para se ter uma ideia, a indústria dos videogames faturou 134 bilhões de dólares anuais em 2018 no mercado global, deixando para trás setores consolidados do entretenimento, como a indústria de Hollywood (Abbade, 2019). Jovens estadunidenses passam em média 22 horas semanais jogando videogames (Alves, 2014). Já no Brasil, jovens adultos gastam em média 15 horas por semana, enquanto adolescentes chegam a passar mais de 19 horas semanais nos jogos eletrônicos. (Gogoni, 2015).

Tendo em vista a relevância desse fenômeno, alguns pesquisadores notaram que o potencial dos videogames poderia ser usado para outros propósitos. Nos anos 1980, acadêmicos da área de interação humano-computador passaram a considerar elementos dos jogos como fontes de ideias para o desenvolvimento de interfaces mais agradáveis. Em virtude disso, deram continuidade às pesquisas até que, nos anos 2000, estes elementos passaram a ser explorados na área de design para experiência do usuário, dentre outros (Deterding, 2012). Mais recentemente, pesquisadores têm explorado o potencial motivacional dos jogos, extraindo seus elementos para aplica-los nos mais diversos contextos. Salas de aula, empresas e até mesmo instituições governamentais estão utilizando elementos de jogos em busca de maior engajamento e envolvimento dos indivíduos. $\mathrm{E}$ esse movimento tem constituído um fenômeno referenciado pelos estudiosos da área como gamificação, termo derivado da palavra de origem inglesa gamification.

Conforme nos explica Formanski (2016, p. 32) a gamificação utiliza mecânicas de jogos em ambientes que não são de jogos. A experiência do jogo, segundo Huizinga (2000), leva ao distanciamento do mundo cotidiano e proporciona uma vivência em um mundo paralelo guiado por regras próprias. A gamificação busca replicar esta experiência e, assim como no jogo, cria um ambiente com elementos atraentes, nos quais existem regras específicas, gerando motivação e envolvimento para estimular os participantes (Brazil, 2017). O propósito da gamificação é o 
engajamento e a participação, para tanto é importante entender e saber aplicar os gatilhos que influenciam os comportamentos em direção aos resultados desejados (Dale, 2014).

Na leitura de Morschheuser, Werder, Hamari \& Abe (2017), a popularização da gamificação tem gerado expectativas promissoras sobre a modernização de processos, entretanto, estes autores alertam que a maioria dos esforços para se gamificar distintos ambientes ainda podem fracassar, devido a dificuldades para projetação e implementação de tais esforços. Frente a esta realidade, este artigo propõe-se a desenvolver diretrizes para gamificação com base na abordagem do design thinking.

Segundo Brown (2017), o design thinking é uma abordagem interdisciplinar, aplicável aos negócios e à inovação social, cujas ferramentas possibilitam que pessoas que não são designers, trabalhem coletivamente seguindo princípios dos pensamentos e processos de design. Dessa maneira, busca-se "estabelecer correspondências entre as necessidades humanas com os recursos técnicos disponíveis considerando as restrições práticas dos negócios” (Brown, 2007, p. 3).

Cardon e Leonard (2010) definem o design thinking como uma abordagem utilizada para estimular a estruturação do pensamento crítico e criativo ao longo da visualização, compreensão e solução de problemas complexos. Neste sentido, Gonsales (2017) ressalta que o design thinking também incentiva o desenvolvimento de novas formas de exploração e solução de problemas seguindo um modelo de pensamento centrado nas necessidades das pessoas.

No design thinking as ideias e soluções são elaboradas junto com os usuários, por meio de processos de cocriação (Funicelli, 2017; Silva, 2014). Moreira (2018) concorda com os demais autores, ao afirmar que o design thinking é uma abordagem centrada no ser humano que integra a interdisciplinaridade com uma nova forma de pensar para encontrar soluções inovadoras. Por fim, Bucker (2015) relaciona o design thinking a uma forma de pensamento:

$\mathrm{O}$ design thinking tem sido estudado como um tipo de pensamento que usa o design como ferramenta de trabalho mental de forma holística, ou seja, uma conformação sistêmica e ampla, que percebe diversos fatores e relações, não somente aquelas atreladas ao produto, como também à sociedade, e particularidade humana (Bucker 2015 apud. Funicelli, 2017. p. 33).

A partir da abordagem colaborativa e das ferramentas que direcionam a equipe a convergir e a divergir, o design thinking estimula a geração de ideias e a criatividade. Boschi (2012, p. 55) diz que o "design thinker utiliza ferramentas diversas para concretizar os princípios de busca por insight e colaboração". 
Neste artigo, argumentamos que o design thinking pode contribuir para as atividades de gamificação por estimular a inovação e também porque ambos têm como foco os usuários. De acordo com Bussarelo (2016), gamificar é algo que requer a compreensão das motivações intrínsecas e extrínsecas das pessoas; já o design thinking visa a solução de problemas para melhorar o bem-estar humano (Vianna, M., Vianna, I., Lucena, \& Russo, 2012). Portanto, as perguntas de pesquisas que guiaram este estudo foram: Como a utilização do design thinking pode contribuir para a geração de ideias criativas focadas nos usuários em atividades de gamificação? E é possível discutir diretrizes para aplicação do design thinking em múltiplos contextos de gamificação?

\section{DESENVOLVIMENTO}

Esta sessão tem início com a descrição dos procedimentos metodológicos, seguida pela discussão de resultados. As etapas do design thinking são apresentadas, depois disso, são explicadas as ferramentas de design thinking a serem aplicadas em diversos contextos de gamificação. Discute-se, então, possíveis diretrizes que poderão guiar processos de gamificação e suas relações com o design thinking. Ao final, parte-se para as considerações finais e sugestões para o desenvolvimento de futuros trabalhos.

\subsection{METODOLOGIA}

De acordo com Brezzon, Miotto e Crivelaro (2005) o método de pesquisa utilizado neste artigo foi o levantamento bibliográfico, o qual incluiu livros, periódicos, revistas, artigos científicos e jornais. A pesquisa realizada foi exploratória, posto que objetivou "proporcionar maior familiaridade com o problema, com vistas a torná-lo mais explícito" (Gil, 2002, p. 41). E a análise da literatura pesquisada baseou-se na interpretação pessoal dos autores, sem informar critérios quantitativos utilizados para avaliação e seleção de trabalhos, caracterizando uma revisão narrativa conforme Rother (2007).

\subsection{DISCUSSÃO DE RESULTADOS}




\subsubsection{ETAPAS DO DESIGN THINKING}

O processo de design thinking é constituído de diferentes etapas, porém diversos autores como Brown (2017), Liedtka \& Ogilvie (2015), Logan (2012) e Vianna et al. (2012) divergem nos modos como definem e denominam quais seriam essas etapas. Por exemplo, Vianna et al. (2012) trabalham com as fases de Imersão, Ideação e Prototipação, já o Design Council (2019) propõe a abordagem do Diamante Duplo, que separa o processo nas fases de Descobrir, Definir, Desenvolver e Entregar. Neste artigo, seguiremos a abordagem de Brown (2017), que considera as fases de Inspiração, Idealização e Implementação.

\subsubsection{INSPIRAÇÃO}

A fase de Inspiração trata da análise do "problema ou da oportunidade que motiva a busca por soluções" (Brown, 2017, p. 16). Nos processos de gamificação, esta fase consiste em conhecer o público do ambiente a ser gamificado e elaborar um briefing, que se trata de um conjunto de instruções a partir das quais a equipe de projeto pode iniciar o trabalho.

Um dos pontos mais importantes do design thinking é a observação. Uma boa solução deve condizer com a realidade de seu público, por isso conhecê-lo bem é primordial. Para isso, não podemos contar apenas com o resultado de pesquisas de mercado ou entrevistas, afinal, muitas vezes as pessoas não sabem expressar quais são suas reais necessidades. É fundamental desenvolver uma conexão profunda com os usuários do ambiente a ser gamificado, ter empatia pelos mesmos e aprender a colocá-los em primeiro lugar (Brown, 2017). Só assim a equipe de desenvolvimento poderá saber quais soluções são adequadas às necessidades e à realidade do público em questão. O caminho mais óbvio seria observar os usuários típicos que se encontram dentro da curva de distribuição normal, à procura de ideias que se adequem ao maior número possível de usuários. Não podemos abrir mão de conhecermos bem este tipo de usuários, porém, soluções realmente inovadoras podem ser encontradas por meio da observação dos comportamentos dos chamados "usuários radicais", que vivem e consomem de formas diferenciadas (Brown, 2017).

Um cuidado com o briefing também é bastante importante, pois, se ele for restrito demais poderá impedir soluções realmente inovadoras, oferecendo um resultado apenas incremental. Já um briefing muito abstrato pode deixar a equipe de desenvolvimento sem saber em que direção 
seguir, muitas vezes oferecendo resultados que não condizem com a real necessidade do público (Brown, 2017).

Para desenvolver a abordagem na prática, Mendonça et al. (2017, p. 15) dizem que "o design thinking envolve diferentes técnicas, mídias e procedimentos de trabalho, que convergem para a visualização do conhecimento, como um processo criado de forma colaborativa". Para tanto, são utilizadas diversas ferramentas que podem auxiliar no desenvolvimento das etapas do design thinking, conforme indicadas por Vianna et al. (2012).

$\mathrm{Na}$ fase de inspiração para gamificação, destacam-se as seguintes ferramentas:

- Reenquadramento: analisa os problemas com relação ao ambiente a ser gamificado sob diferentes perspectivas, desconstruindo crenças e suposições sobre seu público e estimulando os envolvidos a enxergarem os dilemas relacionados sobre diferentes óticas. Consiste na captura de dados do ambiente e público em questão e na transformação destes dados, possibilitando a criação de materiais de sensibilização e de impacto, estimulando a equipe a refletir levantando possíveis questões que não ficaram claras (Vianna et al., 2012).

- Pesquisa desk: é um tipo de pesquisa secundária por ser realizada em fontes seguras da internet já publicadas por terceiros em livros, websites, revistas, artigos entre outros. Este tipo de pesquisa explora as tendências no mundo a respeito do tema discutido e ajuda a equipe a compreender melhor as perspectivas do assunto, trazendo novas áreas de oportunidade a serem exploradas, segundo os autores (Vianna et al., 2012).

- Entrevistas: por meio de uma conversa estruturada por perguntas que podem ser flexibilizadas de acordo com o contexto, mergulha-se no universo dos atores e do assunto tratado. Assim, amplia-se o entendimento a respeito dos comportamentos, pensamentos e sentimentos do público envolvido (Vianna et al., 2012).

- Personas: são arquétipos concebidos a partir de comportamentos observados entre os usuários. Representam seus desejos e necessidades sintetizando suas características mais significativas (Vianna et al., 2012). Pensando no desenvolvimento de personas para a gamificação, é interessante considerar o estudo de Bartle (1996) que divide os jogadores entre quatro perfis: Empreendedores (Achievers), Exploradores (explorers), Socializadores (socializers) e Assassinos 
(Killers), a fim de abranger os variados tipos de comportamento que os usuários poderão demonstrar ao interagirem com cada ambiente gamificado.

\subsubsection{IDEALIZAÇÃO}

É a fase em que o processo é desenvolvido (Brown, 2017), são geradas ideias inovadoras com base nas informações coletadas na fase de inspiração a fim de criar soluções que estejam de acordo com o contexto do usuário final. É importante que essa fase seja realizada por equipes interdisciplinares com grande variedade de perfis, incluindo também no processo aqueles que irão utilizar do produto final (Vianna et al., 2012), no caso os usuários do ambiente gamificado.

Brown (2017) menciona que nas “equipes inteligentes" designers trabalham com psicólogos, etnógrafos, escritores e outros profissionais para garantir que a busca de soluções abarque conhecimentos das mais diversas áreas. Para o autor, esses profissionais precisam apresentar pontos fortes em duas dimensões, o que ele chama de "pessoas em forma de T", apresentando em seu eixo vertical uma competência profunda em sua área de atuação e, no eixo horizontal, a capacidade de colaborar em diferentes disciplinas. Segundo o autor, esta característica dos membros é o que distingue equipes meramente multidisciplinares de equipes genuinamente interdisciplinares. Do ponto de vista da gamificação, é importante que as equipes contem com programadores e game designers capazes de contribuir com outras áreas importantes como a psicologia, o marketing e a antropologia, assim como profissionais dessas outras áreas que tenham conhecimentos em game design e programação.

Nesse sentido, uma das práticas mais importantes no processo de design thinking é a cocriação, ou seja, a produção coletiva de ideias envolvendo não só as equipes de projeto, mas também os usuários finais dos produtos, de maneira a lhes proporcionar as melhores experiências possíveis. Porém, é preciso evitar que as equipes sejam muito grandes, para que não atuem de formas dispersas. Por consequência, deve-se trabalhar com redes interdependentes de pequenas equipes, atuando de maneiras focadas em processos flexíveis e dinâmicos, capazes de dialogar e aprender com os outros grupos (Brown, 2017).

Segundo Vianna et al. (2012), a fase de idealização é iniciada com a equipe do projeto realizando um levantamento de ideias, baseado em todas informações adquiridas na fase da inspiração. Algumas ferramentas que auxiliam nessa fase serão listadas a seguir: 
- Brainstorming: é uma técnica que estimula a criatividade e participação da equipe, coordenada por um moderador que deve deixar os participantes a vontade para expressar o maior número de ideias possíveis sem perder o foco. Para um brainstorming eficaz os autores recomendam que alguns preceitos sejam seguidos, como o não julgamento das ideias, priorizar a quantidade e não a qualidade, estimular perspectivas ousadas e combinar ideias. De acordo com Instituto Educa Digital (2014), as ideias mais promissoras devem ser refinadas depois e passar por um "choque de realidade" sendo confrontadas para analisar sua viabilidade.

- Workshop de cocriação: é um encontro em forma de sessão estruturada em atividades práticas e dinâmicas a fim de estimular a criatividade e a interação da equipe para geração de novas ideias para solucionar o tema em questão. Os autores explicam que novas visões são necessárias e podem surgir a partir de atividades que estimulam a criatividade em momentos de impasse (Vianna et al., 2012).

- Matriz de posicionamento: é uma ferramenta importante para a análise das ideias geradas ao longo do projeto, identificando suas oportunidades e desafios relacionando cada ideia às necessidades das personas. É utilizada para apoiar a tomada de decisão em relação aos passos a serem seguidos (Vianna et al., 2012).

\subsubsection{PROTOTIPAÇÃO}

De acordo com Brown (2017), a prototipação impede que a equipe gaste muito tempo trabalhando em uma ideia final, que pode acabar se mostrando improdutiva. Uma boa equipe trabalha com uma abordagem experimental, elaborando e testando protótipos desde as primeiras fases do projeto. Nas palavras de Brown (2017, p. 17): "Falhe muitas vezes para ter sucesso mais cedo".

Nesta fase, o ambiente de trabalho mostra grande importância durante o processo criativo. Dessa maneira, é importante haver espaço para o desenvolvimento de vários protótipos, como também deixar sempre visíveis os materiais e dados colhidos nas fases de inspiração e idealização, para incentivar a criatividade da equipe (Brown, 2017).

Quando falamos em protótipos sempre pensamos em produtos físicos, modelos feitos em papel, massa de modelar ou até mesmo objetos bem-acabados que se aproximam muito com o produto final. Considerando que a gamificação não é um produto físico, como seria possível 
prototipá-la? Prototipar a gamificação segue o mesmo princípio que a prototipação de qualquer outro objeto intangível, como um serviço ou uma interface. Neste caso, modelos gráficos são bemvindos, tanto digitais quanto feitos à mão em papel. Além disso, encenações e storytelling (narração de histórias) também são excelentes formas de prototipação (Brown, 2017). Segundo o Instituto EducaDigital (2014), os protótipos permitem que o grupo apresente suas ideias e obtenha feedbacks e, assim, proporcionam oportunidades para refiná-las.

No desenvolvimento de uma gamificação, algumas mecânicas criadas frequentemente podem gerar resultados imprevistos. Isto ocorre porque a equipe pode não se atentar a possíveis resultados gerados por determinadas regras que foram criadas. A prototipação possibilitará, portanto, a identificação destas falhas imprevistas (Medeiros, 2013). As seguintes ferramentas podem ser utilizadas com este objetivo:

- Storyboard: é "uma representação visual de uma história através de quadros estáticos, compostos por desenhos, colagens, fotografias ou qualquer outra técnica disponível" (Vianna et al., 2012, p. 135). Para estes autores, prototipar através do storyboard permite a visualização o curso da ideia de solução, encontrar pontos em aberto e refinar o produto final.

- Encenação: pode ser utilizada para testar a interação do usuário com uma máquina, até um simples diálogo ou simulação de uma situação entre pessoas. Vianna et al. (2012) comentam que a encenação ajuda a construir etapas, avaliar serviços e melhorar a experiência. Na encenação, os atores devem simular a situação ou experiência de forma natural e espontânea. Na gamificação, a encenação ajuda a entender a relação dos atores com a experiência, prever seus comportamentos e sentimentos e etapas do processo que podem estar confusas (Ibidem).

\subsection{POSSÍVEIS DIRETRIZES PARA GAMIFICAÇÃO}

Com base nas informações obtidas sobre as intersecções entre os temas discutidos neste artigo, foram elaboradas possíveis diretrizes que objetivam contribuir para a gamificação utilizando conceitos de design thinking. Vale ressaltar que a gamificação de processos específicos não faz parte do escopo deste artigo, sendo as diretrizes - que apresentaremos a seguir - discutidas para serem aplicadas em qualquer ambiente gamificado, desde aplicativos virtuais até salas de aula ou organizações. 
1. Formar equipes interdisciplinares: o processo de gamificação deve envolver profissionais de áreas diversificadas, a fim de que cada profissional possa fornecer contribuições referentes à sua área de formação e que nenhum ponto deixe de ser observado por falta de um profissional com um ponto de vista específico. Como nos lembra Brown (2017), as organizações criativas estão sempre buscando pessoas capazes de colaborar com diferentes disciplinas, porque esta capacidade é o que, de fato, diferencia uma equipe realmente interdisciplinar de uma equipe apenas multidisciplinar. (Brown, 2017, p. 26).

2. Conhecer profundamente seu público e se colocar no lugar dele: gamificar um processo sem conhecer profundamente os seus usuários oferece o risco de criar elementos destoantes da realidade deles, que não propiciem uma mudança real de comportamento ou que incentive atitudes e sentimentos imprevistos e muitas vezes desagradáveis como frustração ou competição excessiva. É fundamental desenvolver uma conexão profunda com os usuários do ambiente a ser gamificado, ter empatia pelos mesmos e aprender a colocá-los em primeiro lugar (Brown, 2017). $\mathrm{Na}$ etapa Inspiração, por meio da entrevista, amplia-se o entendimento a respeito dos comportamentos, pensamentos e sentimentos do público envolvido (Vianna et al., 2012).

3. Envolver o cliente e os usuários finais no processo: para desenvolver soluções alinhadas com a realidade dos usuários é importante que eles participem do processo colaborando com a experiência. A participação do cliente também é fundamental, já que ele definirá os comportamentos que serão incentivados nos usuários através da gamificação. No design thinking as ideias e soluções são elaboradas junto com os usuários, por meio de processos de cocriação (Silva, 2014; Funicelli, 2017). Neste sentido, Vianna et al (2012) explicam que em momentos de impasse novas visões são necessárias e podem surgir a partir de atividades que estimulem a criatividade.

4. Desenvolver soluções que atendam aos perfis de jogadores: além de conhecer as pessoas que irão utilizar o ambiente, é vital ainda conhecer as formas como elas jogam. Nesse ponto, as personas são muito importantes porque consistem em arquétipos que representam os desejos e necessidades dessas pessoas, sintetizando 
suas características mais significativas (Vianna et al., 2012). O processo gamificado deve incluir elementos que satisfaçam os quatro perfis de jogadores citados por Bartle (1996); relembrando - empreendedores, exploradores, socializadores e assassinos - para que o ambiente não se torne desinteressante para as pessoas cujos perfis não foram atendidos.

5. Observar o comportamento de usuários radicais: para soluções inovadoras podemos contar com usuários que não seguem os padrões. Observando o comportamento de jogadores, podemos notar diversos exemplos de comportamentos não esperados, como pessoas que usam jogos de lutas apenas para criarem personagens ou que utilizam os personagens dos jogos de MMO (jogo multijogador maciço online) com o objetivo de desenvolver narrativas. Com uma profunda observação do comportamento desse tipo de jogadores podemos extrair insights que não seriam possíveis apenas se atentássemos para o comportamento de jogadores tradicionais. O caminho mais óbvio seria observar os usuários típicos que se encontram dentro da curva de distribuição normal, à procura de ideias que se adequem ao maior número possível de usuários. Não podemos abrir mão de conhecermos bem este tipo de usuários, porém, soluções realmente inovadoras podem ser encontradas por meio da observação dos comportamentos dos chamados "usuários radicais", que vivem e consomem de formas diferenciadas (Brown, 2017).

6. Investir tempo em prototipação: acrescentar elementos em processos gamificados sem que eles tenham sido testados anteriormente pode expor o projeto ao risco de criar soluções ineficientes, que podem incentivar comportamentos indesejados. A prototipação deve ocorrer assim que surgirem as primeiras ideias, para evitar que se perca tempo trabalhando em soluções que poderão se mostrar inválidas; e, assim, continuar o desenvolvimento do processo até que se obtenha um modelo final para ser testado definitivamente. O uso do storyboard é uma excelente alternativa para prototipação em gamificação porque facilita a visualização da ideia proposta como solução, como também a identificação de aspectos a serem aprimorados no produto final (Vianna et al 2012). A encenação também pode ser 
usada para esta finalidade porque ajuda a construir etapas, avaliar serviços e melhorar a experiência (Ibidem).

\section{CONSIDERAÇÕES FINAIS}

Concluímos que o design thinking oferece muitas possibilidades para contribuições em atividades de gamificação. Afinal, um processo tão recente e inovador requer estratégias igualmente inovadoras para que seus objetivos sejam atingidos. Não há como pensar em gamificação apenas reproduzindo velhos pensamentos, pois atitudes previsíveis levam a resultados previsíveis, não contribuindo para a mudança de comportamento que a gamificação tanto busca.

$\mathrm{O}$ objetivo deste artigo foi encontrar as lacunas em que o design thinking, seus pensamentos, estratégias e ferramentas poderiam contribuir para a gamificação. A discussão concluiu que o ponto de partida para realizar uma gamificação eficiente é conhecer profundamente os usuários do ambiente ou sistema que será gamificado, e para isso, a observação dos mesmos é essencial. Os autores aconselham diferentes ferramentas que podem auxiliar no processo, neste artigo foram abordadas as ferramentas de reenquadramento, pesquisa desk, entrevistas e personas, que são evidenciadas pelas diretrizes $n^{\circ} 2$ : "Conhecer profundamente seu público e se colocar no lugar dele"; $n^{\circ}$ 4: "Desenvolver soluções que atendam aos perfis de jogadores" e $n^{\circ}$ 5: "Observar o comportamento de usuários radicais".

Os autores também salientam a importância das equipes interdisciplinares e da cocriação para o processo, sugerindo o brainstorming, o workshop de cocriação e a matriz de posicionamento como ferramentas para auxiliar o desenvolvimento de ideias e a tomada de decisão. A atenção dedicada às equipes é evidenciada na diretriz n 1 : "Formar equipes interdisciplinares". A diretriz n 3 : "Envolver o cliente e os usuário finais no processo" abrange tanto o processo de conhecer e compreender bem o cliente, quanto o desenvolvimento das equipes de projeto. Por fim, é discutida a importância da prototipação, sendo sugeridas as ferramentas de storyboard e encenação para auxiliar nesta etapa, que é abordada na diretriz no 5 : "Investir tempo em prototipação".

Pensando em trabalhos futuros, sugerimos a realização de análises aprofundadas sobre $\mathrm{o}$ desenvolvimento de personas para gamificação com base nos perfis de jogadores criados por Bartle (1996). Um trabalho sobre a formação de equipes inteligentes para desenvolver processos de gamificação também se mostra pertinente e poderá contribuir para o desenvolvimento desta área de pesquisa. 


\section{AGRADECIMENTOS}

O presente trabalho foi realizado com apoio da Coordenação de Aperfeiçoamento de Pessoal de Nível Superior - Brasil (CAPES) - Código de Financiamento 001.

\section{REFERÊNCIAS}

Abade, J. (2019) Indústria dos videogames bate recordes e fatura US\$ 134 bilhões. Jovem Nerd. RAE-eletrônica Recuperado de https://jovemnerd.com.br/nerdbunker/industria-dosvideogames-bate-recordes-nos-eua-e-fatura-us-43-bilhoes/

Alves, C. (2014) Quantas horas você passa ligado nos games? Estudo revela algumas estatísticas. Purebreak. RAE-eletrônica. Recuperado de https://www.purebreak.com.br/noticias/quantashoras-voce-passa-ligado-nos-games-estudo-revela-algumas-estatisticas/4786.

Bartle, R. (1996). Hearts, clubs, diamonds, spades: players who suit MUDs. Recuperado de http://mud.co.uk/richard/hcds.htm.

Bezzon, L.C., (Org.). (2005). Guia prático de monografias, dissertações e teses: Elaboração e apresentação ( $3^{\mathrm{a}}$ ed.). Campinas, SP: Editora Alínea

Boschi, M. T. (2012). O design thinking como abordagem para gerar inovação: uma reflexão. (Dissertação de Mestrado) - Universidade Anhembi Morumbi, São Paulo, SP, Brasil. Recuperado de http://tede.anhembi.br/tedesimplificado/handle/TEDE/1607.

Brazil, A. L (2017). Gamificação na produção colaborativa de conhecimento e informação. (Tese de Doutorado). Universidade Federal do Rio de Janeiro, RJ, Brasil. Recuperado de http://ridi.ibict.br/bitstream/123456789/948/1/TESE\%20ANDRE\%20BRAZIL\%20PPGCI\% 20IBICT\%20UFRJ\%202017.pdf.

Brown, T. (2017). Design thinking: uma metodologia poderosa para decretar o fim das velhas ideias. [Rio de Janeiro]: Alta Books

Bussarelo, R. I. (2016a). Gamificação em histórias em quadrinhos hipermídia: diretrizes para construção de objeto de aprendizagem acessível (Tese de Doutorado). Universidade Federal de Santa Catarina, Florianópolis, SC. Brasil. Recuperado de: http://www.bu.ufsc.br/teses/PEGC0455-T.pdf.

Busarelo, R. I. (2016b) Gamification: princípios e estratégias. [São Paulo]: Pimenta Cultural

Dale, S. (2014) Gamification: Making work fun, or making fun of work? Business Information Review. $\quad$ p. 82-90. Recuperado de: https://www.researchgate.net/publication/270723662_Gamification_Making_work_fun_or_ making_fun_of_work . 
Design Council, (2019, jul, 9) News \& opinion. Disponível em: https://www.designcouncil.org.uk/news-opinion/design-process-what-double-diamond.

Deterding, S. (2012) Gamification: Designing for motivation. Interactions, (v. 19, n. 4, p.14-17). Association for Computing Machinery (ACM).

Formanski, F. N. (2017) Aplicabilidade da gamificação no contexto empresarial (Dissertação de Mestrado) Programa de Pós-graduação em Engenharia e Gestão do Conhecimento, Universidade Federal de Santa Catarina, Florianópolis, 2016. Recuperado de http://btd.egc.ufsc.br/?p=2054.

Funicelli, V. B. (2017) Design thinking como metodologia de inovação e colaboração. (Dissertação de Mestrado) Programa de Estudos Pós-Graduados em Tecnologia da Inteligência e Design Digital, Pontifícia Universidade Católica de São Paulo, São Paulo, SC, Brasil. Recuperado de https://tede2.pucsp.br/handle/handle/20692?mode=full.

Gil, A.C. (2002) Como elaborar projetos de pesquisa. 4. ed. [São Paulo]: Atlas.

Gogoni, R. (2015) Brasil Gamer: 82\% dos jovens e adultos jogam videogames. Meiobit, RAEeletrônica Recuperado de https://meiobit.com/328936/brasil-pesquisa-npd-82-por-centopopulacao-entre-13-59-anos-jogam-entre-pcs-consoles-mobile-e-portateis/.

Huizinga, J. (2000) Homo Ludens. Tradução de João Paulo Monteiro. 4a. Ed. [São Paulo]: Ed. Perspectiva.

Instituto EducaDigital, (2014) Design thinking para educadores. Recuperado de https://www.dropbox.com/sh/hgnb8w3kbz1nhef.

Liedtka, J., \& Ogilvie, T. (2015). A magia do design thinking: um kit de ferramentas para o crescimento rápido da sua empresa. São Paulo: HSM

Logan, R. K. (2012) Design thinking, Strategic Foresight, Business Model Generation and Biology: A Mashup. Conference paper for use in workshops at MaRS and in the Think Tank course at OCAD University Ontario College of Arts and Design University Recuperado de: https://www.researchgate.net/publication/267037971.

Medeiros, M. Fo, (2013) A importância da prototipação no design de games. SBC-Proceedings of SBGames. RAE-eletrônica. Recuperado de http://www.sbgames.org/sbgames2013/proceedings/artedesign/37-dt-paper.pdf.

Mendonça, M. C., Almeida, F. S., Fialho, F.A.P., Schimiegelow, S. S., \& Souza, R.P.L, (2017). Design thinking, mídia, conhecimento e inovação: reflexões sobre uma atividade didática aplicando o desenho da persona e o mapa da jornada do usuário. Anais do Congresso CIKI Internacional do Conhecimento e Inovação, Foz do Iguaçu, Paraná, Brasil, 7. Recuperado de http://proceeding.ciki.ufsc.br/index.php/ciki/article/view/170/60.

Morschheuser, B., Werder, K., Hamari, J. \& Abe, J. (2017). How to gamify? A method for designing gamification. In Proceedings of the 50th Annual Hawaii International Conference 
on System Sciences. University of Hawai'i at Manoa,. Recuperado de https://pdfs.semanticscholar.org/2c4f/c8c41f31dfbac69bac7079448da26da33049.pdf.

Neto D. M. L. (2016) O Jogo Real de Ur. Superinteressante. RAE-eletrônica. Recuperado de: https://super.abril.com.br/comportamento/o-jogo-real-de-ur/.

Rother, E. T. (2007). Revisão sistemática X revisão narrativa. Acta Paulista de

Enfermagem, 20(2), v-vi. Recuperado de: https://dx.doi.org/10.1590/S0103-21002007000200001

Vianna, M., Vianna, I. Adler, I. K., Lucena \& B., Russo, B. (2012). Design thinking: inovação em negócios. [Rio de Janeiro]: MJV Press. E-book. Recuperado de https://cdn2.hubspot.net/hubfs/455690/Ofertas/E-books/Arquivos/livro-design-thinkingbusiness-innovation.pdf. 